\title{
Development of a Radial Basis Function Neural Network Model to Predict High b-value Diffusion MR Signals of the Prostate
}

\author{
Gokhan ERTAS
}

\begin{abstract}
Submitted: 27/06/2019 Accepted: 10/05/2020
Abstract: In prostate cancer detection and diagnosis, high $b$-value diffusion MR signals of the prostate tissues are of great concern. MR scanners and computation methods face difficulties in obtaining these signals. This study aims development of a neural network model to predict the MR signal amplitudes at high $b$-values from the amplitudes at low $b$-values. Synthetic diffusion MR signals are generated using a kurtosis model for noise-free and noisy conditions considering nine $b$-values: the low $b$-values are $0,50,250,500,750 \mathrm{~s} / \mathrm{mm}^{2}$ and the high $b$-values are 1000,1250,1500, 2000s/ $\mathrm{mm}^{2}$. Four radial basis functions neural networks (RBF-NN) connected in parallel are designed to accept the signal amplitudes at low $b$-values and to provide signal amplitudes at the high $b$-values. RBF-NNs housing altered number of neurons with radial basis functions attributing different widths in the hidden layers of the networks are analyzed. Learning and prediction performances of the NNs are assessed from training and testing datasets. For the noise-free condition, RBF-NNs reveal perfect predictions $(r=1.000)$ with very good learnings $\left(M S E=0.76-0.02 \times 10^{-6}\right)$. For the noisy conditions, the RBF-NNs achieve moderate to strong predictions ( $r=0.981-0.463$ ) with good learnings ( $\left.M S E=0.32-10.33 \times 10^{-3}\right)$. Prediction performance reduces as the level of noise and/or targeted high $b$-value increases. RBF-NNs facilitate prediction of high $b$-value diffusion MR signals of the prostate by requiring no diffusion signal decay function, optimization algorithm or initial/boundary values for the optimization algorithm. They may be quite functional in accurate voxel-wise generation of high $b$-value MR images for early detection and diagnosis of prostate cancer. Further prospective studies are needed to justify the potential benefits in clinical practice.
\end{abstract}

Keywords: Prostate, diffusion signal, neural network, prediction

\section{Introduction}

Diffusion-weighted imaging (DWI) is a non-invasive magnetic resonance (MR) imaging technique that is gaining increased acceptance in characterization of human tissue for early detection and diagnosis of cancer [1]. The technique relies on tracing microscopic mobility of water molecules influenced by cell density, membrane integrity, microstructure, perfusion and diffusion heterogeneity within the tissues. A diffusion MR signal is established from the results of mobility measurements taken for a set of diffusion weightings called $b$-values [2]. Theoretically, a specific $b$-value is obtained by adjusting the strength, duration and spacing of the pulsed gradients established by the MR scanner [3]. Increased gradient amplitude or duration or widened interval between gradient pulses results in a high $b$-value. However, the acquisition of a high $b$-value diffusion signal with acceptable levels of noise is quite challenging due to limitations of the current MR scanner hardware [4]. Exposure of living tissues to a high $b$-value may also lead to some annoying consequences such as mechanical vibration artifacts superimposed on the diffusion signals [5]. Despite these limitations, high $b$-value diffusion MR signals are of great concern in detection and diagnosis of prostate cancer [6]. Computed DWI offers good quality high $b$-value MR signals by eliminating noise and artifacts [7]. It involves extrapolation of the low $b$-value diffusion signal acquired by the MR scanner to obtain the high $b$-value signal for the targeted tissue. The extrapolation process consists of steps that should be performed with utmost

${ }^{1}$ Department of Biomedical Engineering, Yeditepe University, Istanbul,Turkey; ORCID ID: 0000-0002-3331-9152

*Corresponding AuthorEmail: gokhan.ertas@yeditepe.edu.tr care. First, a diffusion signal decay model should be selected appropriately. Next, the model should be fitted to the low $b$-value diffusion signal by intensive mathematical calculations or excessive computations performed using a properly selected optimization algorithm with well-adjusted initial values and/or boundaries of the model parameters. Following the fitting, estimates of the model parameters obtained should be used to compute the signal amplitude for a given high $b$-value. Currently, computed DWI is pronounced with a monoexponential decay model due to its simplicity and ease of fitting [8]. However, it has been reported that the monoexponential decay model offers a limited depiction of the diffusion signal decay for the prostate tissue and a more realistic depiction can be offered by a kurtosis model $[9,10]$. The kurtosis model relies on a monoexponential model modified to include a kurtosis term that reflects both the Gaussian and the non-Gaussian diffusion of water within different prostate tissues [11]. The model inherently requires acquisition of MR signals with both low and high $b$-values that avoids its use in computed DWI. There is need for an alternative method that facilitates extrapolation of the low $b$-value MR signal to predict the amplitudes of the signal for high $b$-values without requiring any pre-defined signal decay model for the prostate.

As artificial neural networks offer unique solutions to highly complex problems in many areas, they have recently been used in the processing of diffusion MR signals. Multilayer perceptron based neural network models are proposed to approximate complicated diffusion signal decay functions even in the presence of noise $[12,13]$. In contrast, the current study introduces a neural network model based on radial basis function neural networks to predict high $b$-value diffusion MR signals of the prostate tissue. 


\section{Materials and Methods}

\subsection{Diffusion MR Signals of the Prostate Tissue}

Synthetic diffusion MR signals of the human prostate gland are generated for both the tumorous tissue and the healthy tissue using a kurtosis model and Monte Carlo trials. Let $s_{b}$ be the diffusion signal amplitude obtained for a specific diffusion weighting determined by a $b$-value and let $s_{0}$ be the signal amplitude attained with no diffusion weighting (i.e., $b=0 \mathrm{~s} / \mathrm{mm}^{2}$ ). The kurtosis model expresses the attenuated diffusion signal by kurtosis analysis [11]:

$$
s_{b} / s_{0}=\exp \left(-b D_{a p p}+\frac{1}{6} b^{2} D_{a p p}^{2} K_{a p p}\right)
$$

Here, $D_{a p p}$ is the kurtosis diffusion coefficient and $K_{a p p}$ is the apparent diffusional kurtosis with no unit. $D_{a p p}$ and $K_{a p p}$ value pairs are quite variable for the prostate tissue; however, higher $D_{a p p}$ and lower $K_{a p p}$ value pairs are conveyed for the healthy tissue and lower $D_{a p p}$ and higher $K_{a p p}$ value pairs are mentioned for the tumorous tissue $[10,14-19]$. In the literature, the only study that applies noise correction on signals states that on mean (SD), $D_{a p p}=$ $2.05(0.36) \times 10^{-3} \mathrm{~mm}^{2} / \mathrm{s}$ and $K_{a p p}=0.64(0.12)$ for the healthy prostate tissue whereas $D_{a p p}=1.53(0.45) \times 10^{-3} \mathrm{~mm}^{2} / \mathrm{s}$ and $K_{a p p}=$ $0.84(0.22)$ for the tumorous prostate tissue from signals acquired at $b$-values of $0,50,250,500,750,1000,1250,1500$ and 2000 $\mathrm{s} / \mathrm{mm}^{2}$ by a $3 \mathrm{~T}$ MR scanner [16]. In the current study, considering these $D_{a p p}$ and $K_{a p p}$ value pairs and the set of $b$-values, Monte Carlo trials are performed to generate one thousand noise-free diffusion signals for tumorous and for healthy prostate tissues by solving Eq. 1. The noisy forms of the signals are obtained by adding Rician noise at four different signal-to-noise ratios (SNR) of 10, 20, 40 and 80 that are commonly experienced in clinical practice [20]. SNR is defined as the ratio of the normalized diffusion signal amplitude obtained without any diffusion weighting to the standard deviation of the noise [21] and the Rician noise is simulated by incorporating two Gaussian noise terms with the same noise standard deviation [22].

\subsection{Neural Network Model to Predict Diffusion MR Signals}

The neural network model developed in the current study consists of four radial basis function neural networks (RBF-NN) connected in parallel as presented in Fig. 1. The input for all the networks is the low $b$-value signal amplitude vector, $\boldsymbol{s}_{\text {low }}$ that comprises the amplitudes of a diffusion MR signal at five low $b$-values of 0,50 , 250,500 and $750 \mathrm{~s} / \mathrm{mm}^{2}$. The output of each network is the predicted amplitude of the diffusion MR signal by the network at a certain high $b$-value, $\tilde{s}_{\text {high }}$. High $b$-values considered in the current study are $1000,1250,1500$ and $2000 \mathrm{~s} / \mathrm{mm}^{2}$ and therefore RBF-NN 1, RBF-NN $2, \mathrm{RBF}_{2} \mathrm{NN}_{3}$ and RBF-NN $\mathrm{N}_{4}$ respectively output $\tilde{s}_{1000}, \tilde{s}_{1250}, \tilde{s}_{1500}$ and $\tilde{s}_{2000}$.

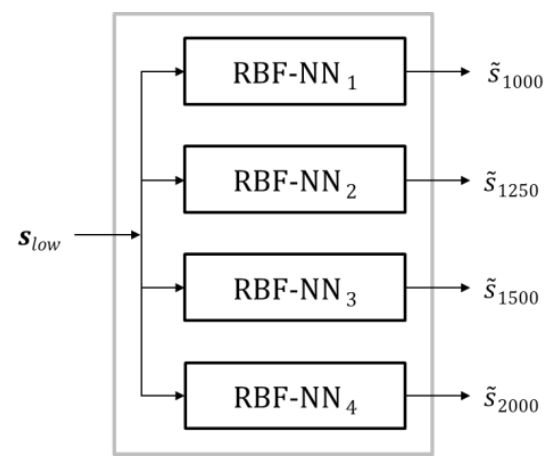

Fig. 1. The neural network model introduced.

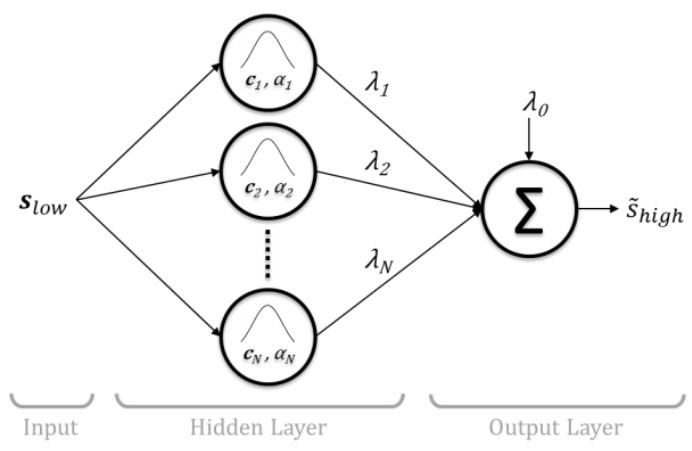

Fig. 2. Architecture of an RBF-NN in the model.

All the networks are equipped with one hidden and one output layers connected in cascade with the same architecture seen in Fig. 2. The output layer is fitted with a single neuron while the hidden layer houses many neurons. Each neuron in the hidden layer owns a Gaussian basis function giving an output by

$$
\varphi_{i}\left(\boldsymbol{s}_{\text {low }}\right)=\exp \left(-\frac{\left\|\boldsymbol{s}_{\text {low }}-\boldsymbol{c}_{i}\right\|}{1.44 \alpha_{i}^{2}}\right)
$$

Here, $\alpha_{i}$ and $\boldsymbol{c}_{i}$ are respectively the width and the center vector of the basis function for the $i$-th neuron in the hidden layer and $\|$.$\| denotes the Euclidean norm. The neuron at the output layer$ reveals the predicted value of the diffusion signal amplitude for a specific high $b$-value using

$$
\tilde{s}_{\text {high }}=\lambda_{0}+\sum_{i=1}^{N} \lambda_{i} \varphi_{i}\left(\boldsymbol{s}_{\text {low }}\right)
$$

here $\lambda_{0}$ is the bias for the neuron in the output layer and $\lambda_{i}$ is the weight of the $i$-th output of the hidden layer to the neuron in the output layer $(i=1,2,3, \ldots, N) . N$ represents the total number of neurons in the hidden layer. In the current work, numerous RBFNNs are assembled with $\alpha$ varying from 0.2 to 2.0 with an increment of 0.2 and $N$ ranging from 4 to 103. This range guarantees accurate assessment of the highest network performance possible.

\subsection{Neural Network Training and Testing}

Among the synthetic diffusion MR signals generated, $60 \%$ of the signals are assigned to training dataset while the remaining $40 \%$ of the signals are assigned to testing dataset randomly. This process is performed ten times resulting in ten randomly assigned training and testing dataset pairs.

The networks assembled are trained with the training datasets by following a two-phase strategy. In the first phase, a predefined number of neurons is populated in the hidden layer and the widths of the Gaussian basis functions for the neurons are all set to a predefined value. The center for each basis function is determined by performing orthogonal least-squares learning [23]. In the second phase, the weights for the outputs of the hidden layer and the bias for the output layer are found by building the system equations and solving the equations using singular value decomposition [24]. Following training, the networks are tested with the relevant testing datasets.

\subsection{Performance Assessment}

The learning performances of the neural networks during training are assessed by mean squared error, MSE that gives the average squared difference between the ground truth and the predicted amplitudes of the diffusion signals for a specific high $b$-value. A lower MSE value denotes better learning for a network [25]. The prediction performances of the networks are assessed during training and testing using Pearson correlation coefficient where $r$ 


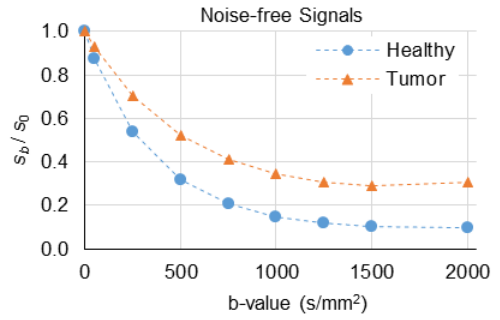

(a)

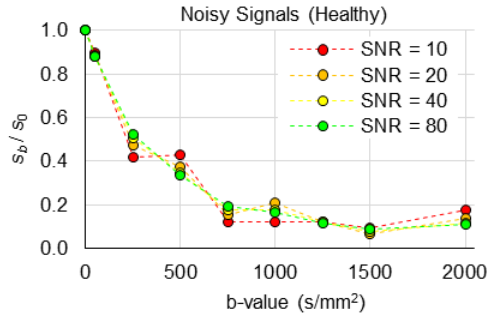

(b)

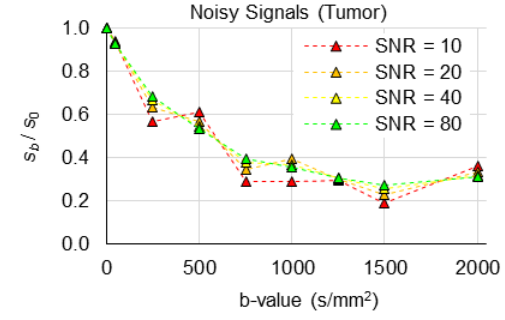

(c)

Fig. 3. Representative (a) noise-free and (b, c) noisy diffusion MR signals.

reveals the linear association strength between the ground truth and the predicted amplitudes of the diffusion signals for a particular high $b$-value. $r$ takes values ranging from -1 (perfect negative association) to +1 (perfect positive association). According to the guidelines of Evans [26], interpretation definitions for the absolute value of $r$ are as follows: 0-0.19: very weak, 0.20-0.39: weak, 0.400.59 : moderate, $0.60-0.79$ : strong and $0.80-1.00$ : very strong. Among the neural networks performing diffusion signal amplitude predictions for the same high $b$-value, the neural network that achieves the highest $r$ with the lowest number of neurons in its hidden layer is recognized as the optimal neural network. Optimal neural networks are identified individually for the noise-free and the noisy conditions.

\section{Results}

Fig. 3a shows representative noise-free diffusion MR signals for the healthy and the tumorous tissues of the prostate generated respectively using $D_{a p p}=2.05 \times 10^{-3} \mathrm{~mm}^{2} / \mathrm{s}$ and $K_{a p p}=0.64$, and $D_{a p p}=1.53 \times 10^{-3} \mathrm{~mm}^{2} / \mathrm{s}$ and $K_{a p p}=0.84$. Noisy forms of the signals obtained by adding Rician noise to the noise-free signals at the four different signal-to-noise ratios (SNR) are shown in Fig. 3b-c (Each tissue is mimicked by five diffusion MR signals considering one noise-free and four noisy conditions).

A total of 10000 diffusion MR signals are synthetically generated in five two-thousand signal sets for the noise-free and four noisy conditions. Of each two-thousand signal set, 1200 signals are randomly assigned to the training dataset and the remaining 800 signals are dedicated to the testing dataset. This process is repeated ten times leading to a total of fifty training and testing dataset pairs used to train and test a total of 1000 radial basis function neural networks (RBF-NN) housing one-hundred different numbers of the neurons $(N=4,5,6, \ldots, 103)$ with ten different basis function widths $(\alpha=0.2,0.4,0.6, \ldots, 2.0)$ in the hidden layer.

Fig. 4 shows the performances of the RBF-NNs in the model developed to predict diffusion signal amplitudes at the high $\quad b$ - values for the noise-free condition. Accordingly, the optimal neural networks identified are as listed in Table 1. The networks do all reveal the perfect positive association $(r=1.000)$ presenting that the signal amplitudes predicted are almost equal to the ground truth signal amplitudes at all high $b$-values targeted (i.e. 1000, 1250,1500 and $2000 \mathrm{~s} / \mathrm{mm}^{2}$ ). However, an increased number of neurons in the hidden layer of the networks is needed to guarantee the perfect positive association. Average squared differences between the ground truth and the predicted amplitudes of the diffusion signals from the training datasets for the networks are quite low $\left(M S E=0.76-0.02 \times 10^{-6}\right)$ illustrating very good learning for all the networks.

Performances of the RBF-NNs in the model developed to predict diffusion signal amplitudes for the noisy conditions are seen in Fig. 5. Optimal neural networks identified are as given in Table 2. For all SNRs considered, the networks do all reveal positive association but with varying degrees $(r=0.981-0.463)$ and varying average squared differences $\left(M S E=0.32-10.33 \times 10^{-3}\right)$. Association strength decreases as SNR decreases and/or target high $b$-value increases. For $\mathrm{SNR}=80$, very strong associations are observable $(r=0.981-0.824)$. The association strengths is lowering as SNR reduces to 40 and is being strong as targeted $b$-value is increased to $2000 \mathrm{~s} / \mathrm{mm}^{2}$ ( $\left.r=0.946-0.691\right)$. As SNR is decreased further to 20 , the association strengths lower further and becoming moderate when targeted $b$-value is set to $2000 \mathrm{~s} / \mathrm{mm}^{2}(r=0.848-0.563)$. When SNR is set to its lowest value of 10 , the association strengths gain their lowest values but the association strength is still moderate when the targeted $b$-value is fixed to $2000 \mathrm{~s} / \mathrm{mm}^{2}(r=0.682-0.463)$. Fig. 6 and Fig. 7 illustrate the tendencies of the prediction and the learning performances offered by the optimal networks with respect to the SNRs and the high $b$-values studied. For a specific SNR, the learning and the prediction performances decrease as the high $b$-value targeted increases; however, increase in SNR leads to better learning and prediction performances regardless of the high $b$-value targeted.
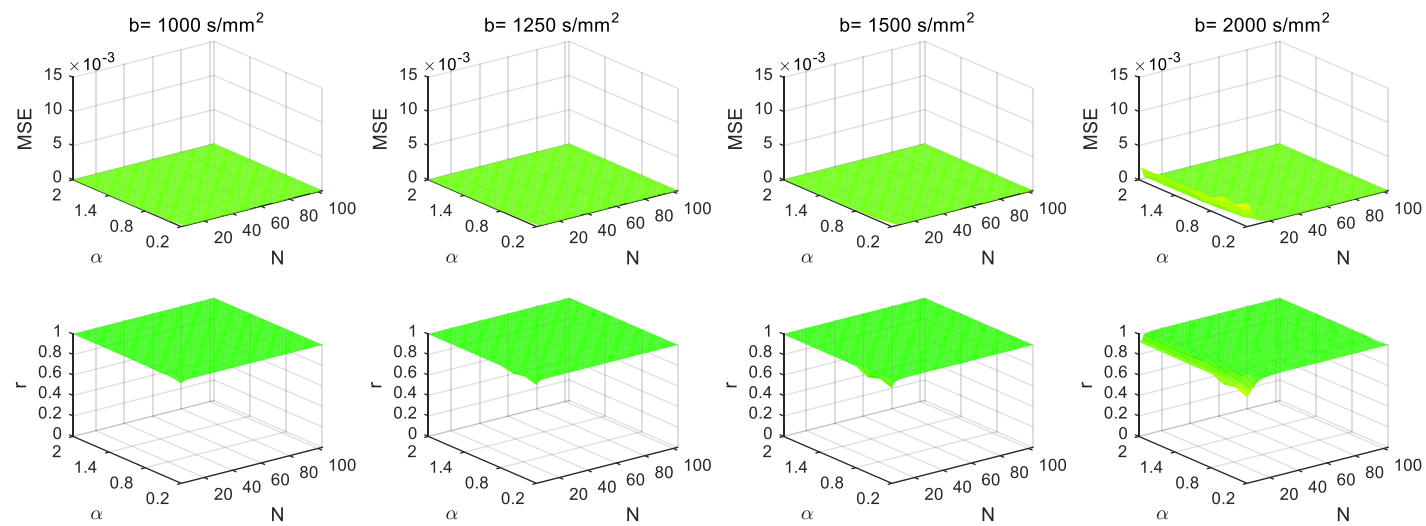

Fig. 4. Performances of the neural networks for the noise-free condition. 

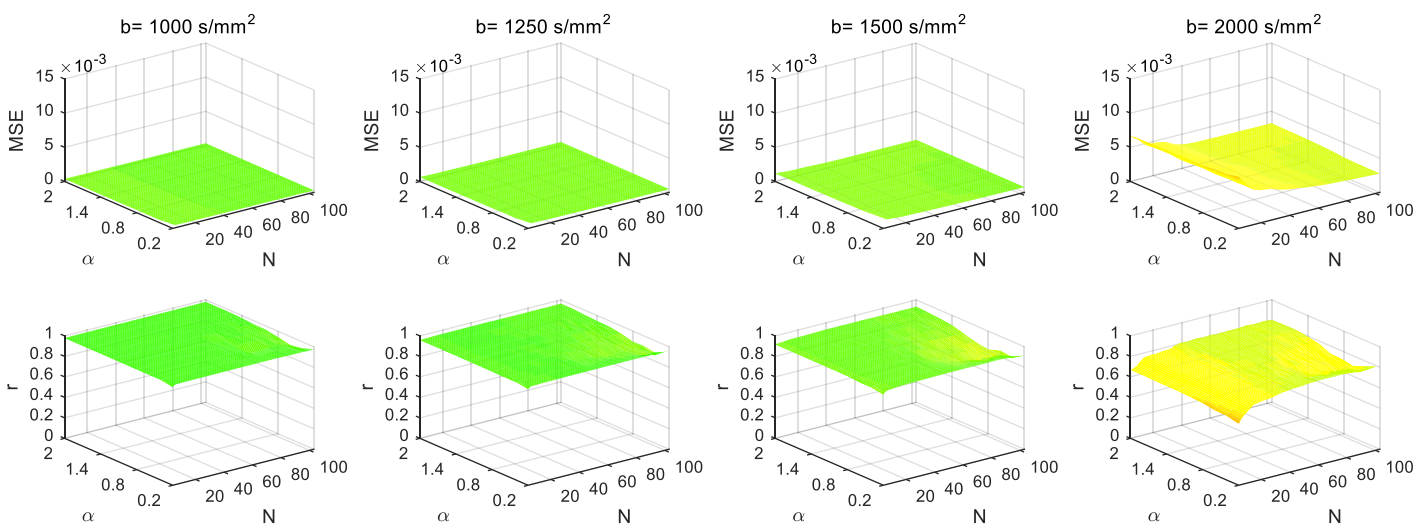

(a)
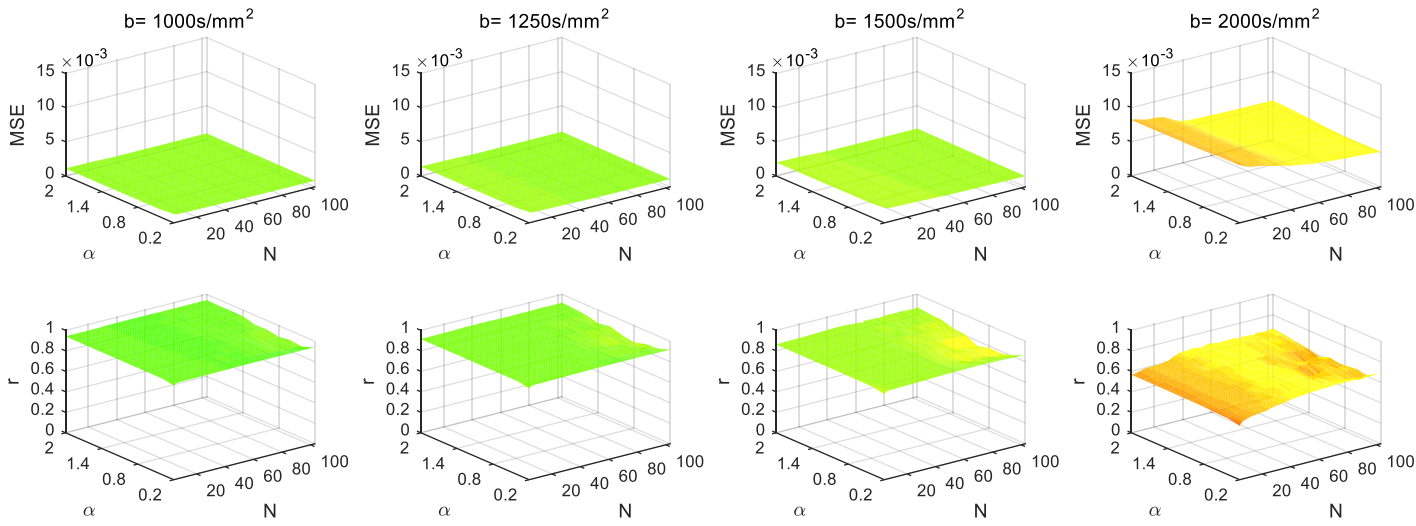

(b)
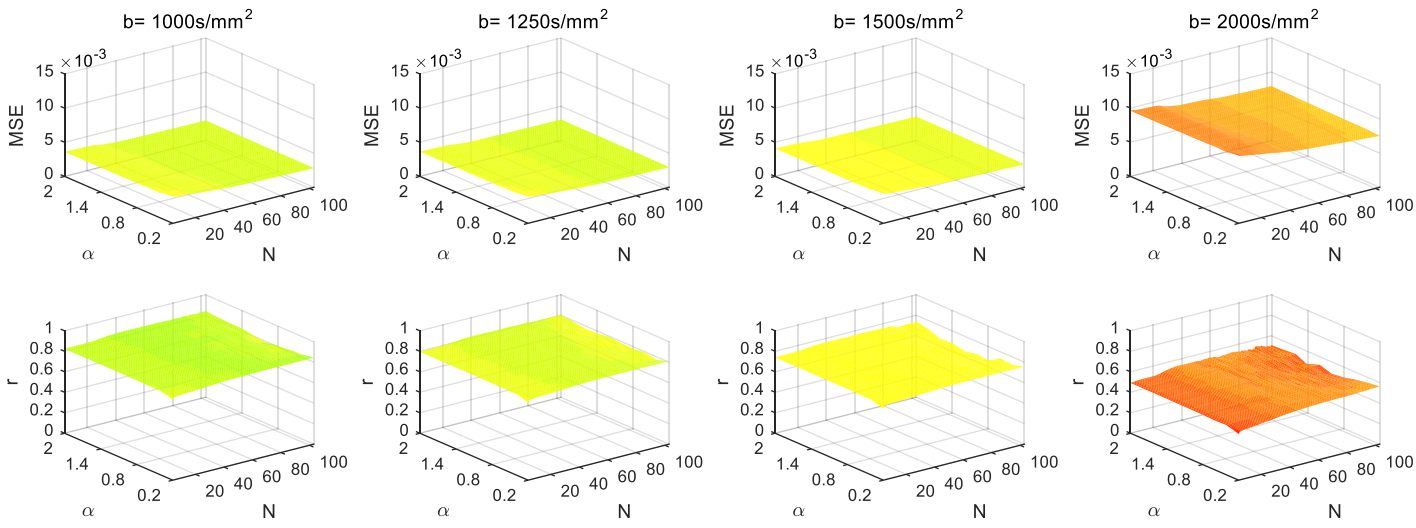

(c)
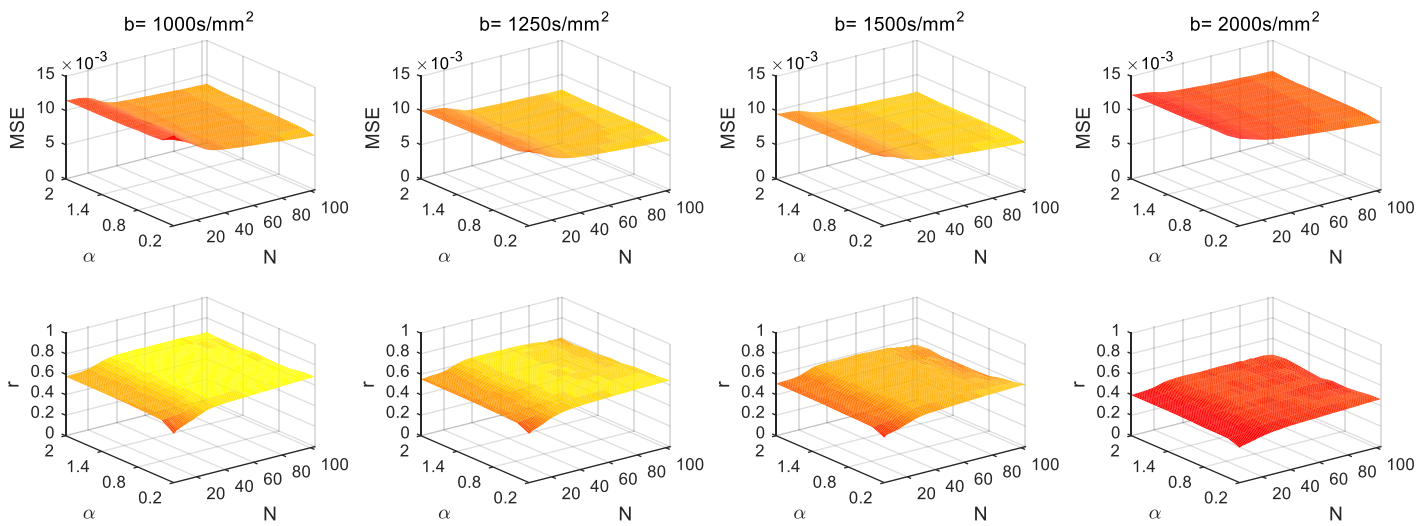

(d)

Fig. 5. Performances of the neural networks for the noisy condition possessing (a) $S N R=80$, (b) $S N R=40$, (c) $S N R=20$ and (d) $S N R=10$. 
Table 1. Optimal neural networks for the noise-free condition

\begin{tabular}{ccccc}
\hline $\begin{array}{c}\text { High } \boldsymbol{b} \text {-value } \\
\left(\mathbf{s} / \mathbf{m m}^{\mathbf{2}}\right)\end{array}$ & \multicolumn{4}{c}{ Optimal Neural Network Attributes } \\
\hline 1000 & $\boldsymbol{r}$ & $\boldsymbol{N}$ & $\boldsymbol{\alpha}$ & $\boldsymbol{M S E}\left(\times \mathbf{1 0}^{-\mathbf{6}}\right)$ \\
\hline 1250 & 1.000 & 5 & 1.0 & 0.71 \\
1500 & 1.000 & 8 & 1.4 & 0.76 \\
2000 & 1.000 & 13 & 1.0 & 0.43 \\
\hline
\end{tabular}

Table 2. Optimal neural networks for the noisy condition

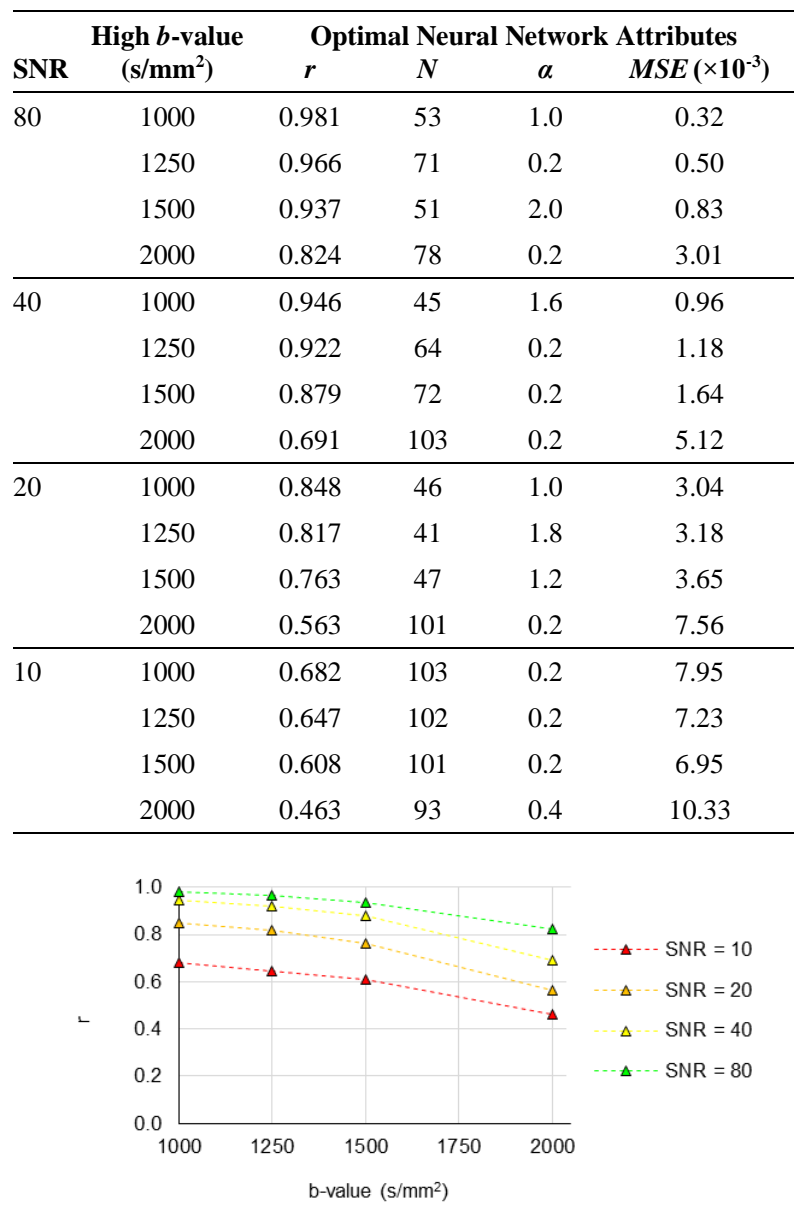

Fig. 6. Prediction performances of the optimal neural networks.

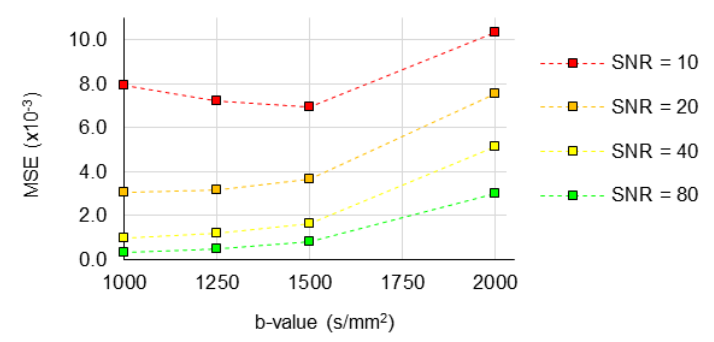

Fig. 7. Learning performances of the optimal neural networks.

\section{Conclusion}

A neural network model has been developed to predict the normalized signal amplitudes of diffusion MR signals of prostate tissues at high $b$-values from the amplitudes acquired at low $\quad b$ values using diffusion weighted imaging (DWI). In the current implementation, the low $b$-values considered are 0,50, 250, 500 and $750 \mathrm{~s} / \mathrm{mm}^{2}$ while $1000,1250,1500$ and $2000 \mathrm{~s} / \mathrm{mm}^{2}$ are the four high $b$-values targeted. Additionally, the model consists of four radial basis function neural networks (RBF-NN) connected in parallel. The networks are all equipped with one hidden layer and one output layer and accept the low $b$-value signal amplitudes as the input. The hidden layer performs a nonlinear transform of the input, and the output layer is a linear combiner mapping the nonlinearity into a new space giving the predicted signal amplitude at a particular high $b$-value. A huge number of RBF-NNs housing altered number of neurons with radial basis functions attributing different widths are tested on prostate diffusion MR signals generated synthetically using a kurtosis model and Monte Carlo trials for noise-free and noisy conditions. In the absence of noise, an RBF-NN may provide perfect predictions for the signal amplitudes at all high $b$-values; however, to assure this performance, it is needed to increase the number of neurons in the hidden layer of the network while the high $b$-value targeted increases. RBF-NNs have very good learning capability for the noise-free signals. For the noisy condition, prediction performance by an RBF-NN reduces while the level of noise increases. In addition to this, the prediction performance also reduces while the high $b$-value targeted increases for a specific noise level. However, outstanding prediction performances are attainable by the RBF$\mathrm{NN}$ for all high $b$-values for low noise levels (i.e. SNR>40). On the other hand, the learning performance of an RBF-NN deteriorates when the level of noise increases and/or the high $b$ value targeted increases.

There are some limitations of the current study. The diffusion MR signals are synthetically generated data mimicking the tissues within the peripheral zone of the prostate only. The performances of the networks reported may be debatable for the clinical data and may also differ for the tissues within the central and the transitional zones of the prostate (For these zones, DWI inherently suffers from a reduced tumor detection performance [27]). The noisy forms of the diffusion signals are produced with Rician noise that converges to Gaussian noise as SNR increases. The Gaussian basis functions within the RBF-NNs might cope with the Gaussian noise better and therefore, the networks could predict signal amplitudes at high $b$ values better as SNR increases. The RBF-NNs are trained using $60 \%$ of the signals and tested with the remaining $40 \%$ of the signals among the synthetic diffusion MR signals generated. These percentages are determined with utmost care after intensive experiments on the entire dataset. Utilization of different percentage values may lead to overtraining or undertraining problems resulting in unrealistically high or low overall prediction performances. The center for each basis function within the RBFNNs is determined by performing orthogonal least-squares learning that offers a simple and efficient means for fitting the networks while overcoming numerical ill-conditioning [23]. However, the use of "recursive" orthogonal least-squares learning may lead to acceptable performances with significant reductions in the number of neurons in the hidden layer of the networks [28]. The widths of basis functions in an RBF-NN are all set to a single value that delivers universal extrapolation capability for the networks, but radial basis functions with dedicated widths might improve the performances of the networks further. The optimal RBF-NNs attributing larger radial basis function widths provide smoother signal extrapolations but the networks with smaller widths might not be generalized well.

To the best of our knowledge, the current work is the first study on the use of neural networks to predict high $b$-value diffusion MR signals. The neural network based solution introduced involves neither a signal decay function nor an optimization algorithm; moreover, signal predictions are performed quite fast once the learning of the neural network is accomplished. Therefore, it may offer less computational complexity and shorter computation time 
than the computed DWI. A comparative work on the benefits of each solution is left as future work. On the other hand, the neural network based solution introduced consists of RBF-NNs connected in parallel that are fed by the signal amplitudes acquired at low $b$-values. Alternatively, the RBF-NNs may be connected in cascade so that the signal amplitude at a particular high $b$-value predicted by an RBF-NN can be supplied with the predictions by the previous networks and the signal amplitudes at low $b$-values to the input of the following network. This setup will iteratively increase the degrees-of-freedom for the solution and may improve the accuracy of the predictions especially for large high $b$-values and for higher noise levels, consequently. This issue is left for future work. Despite these, diffusion MR signals from the tumorous and the healthy tissues of the prostate are solely considered for the current study. Whereas DWI performed at clinical practice reveals diffusion MR signals of the tissue volumes of the prostate that may be mixtures of tumorous and healthy tissues. Testing and validation of the introduced model on such volumes will be aimed in future work.

In conclusion, radial basis function neural networks may facilitate the prediction of high $b$-value diffusion MR signals of the prostate without requiring any diffusion signal decay function, optimization algorithm or initial/boundary values for the optimization algorithm. These networks may also be quite functional in accurate voxel-wise generation of high $b$-value MR images of the whole prostate tissue in detection and diagnosis of prostate cancer. Further prospective studies are needed to justify the potential benefits in clinical practice.

\section{References}

[1] L. Tang and X.J. Zhou, "Diffusion MRI of cancer: From low to high b-values," J Magn. Reson. Imaging, vol. 49, no. 1, pp. 23-40. Jan. 2019.

[2] A.R. Padhani, G. Liu, D.M. Koh, et al. "Diffusion-weighted magnetic resonance imaging as a cancer biomarker: consensus and recommendations," Neoplasia, vol. 11, no. 2, pp. 102-125, Feb. 2009.

[3] E.H. de Figueiredo, A.F. Borgonovi and T.M. Doring, "Basic concepts of MR imaging, diffusion MR imaging, and diffusion tensor imaging," Magn Reson Imaging Clin N Am., vol. 19, no. 1, pp. 1-22, Feb. 2011.

[4] D.M. Koh, M. Blackledge, A.R. Padhani, et al. "Whole-body diffusion-weighted MRI: tips, tricks, and pitfalls," AJR Am J Roentgenol., vol. 199, no. 2, pp. 252-262, Aug. 2012.

[5] M.M. Berl, L. Walker, P. Modi, et al. "Investigation of vibrationinduced artifact in clinical diffusion-weighted imaging of pediatric subjects," Hum. Brain Mapp., vol. 36, no. 12, pp. 4745-57, Dec 2015.

[6] M.H. Maurer and J.T. Heverhagen, "Diffusion weighted imaging of the prostate-principles, application, and advances," Transl. Androl. Urol., vol. 6, no. 3, pp. 490-498, Jun. 2017.

[7] Y.R. Ueno, T. Tamada, S. Takahashi, et al. "Computed diffusionweighted imaging in prostate cancer: basics, advantages, cautions, and future prospects," Korean J Radiol., vol. 19, no. 5, pp. 832-837, Sep. 2018.

[8] M.D. Blackledge, M.O. Leach, D.J. Collins, et al. "Computed diffusion-weighted MR imaging may improve tumor detection," Radiology, vol. 261, no. 2, pp. 573-581, Nov. 2011.

[9] L.N. Mazzoni, S. Lucarini, S. Chiti, et al. "Diffusion-weighted signal models in healthy and cancerous peripheral prostate tissues: Comparison of outcomes obtained at different b-values," J. Magn. Reson. Imaging, vol. 39, no. 3, pp. 512-518, Mar. 2014.

[10]Y. Mazaheri, A.M. Hötker, A. Shukla-Dave, O. Akin, H. Hricak, "Model selection for high b-value diffusion-weighted MRI of the prostate," Magn. Reson. Imaging, vol. 46, pp. 21-27, Feb. 2018.
[11]J.H. Jensen, J.A. Helpern, A. Ramani, et al. "Diffusional kurtosis imaging: the quantification of non-Gaussian water diffusion by means of magnetic resonance imaging," Magn. Reson. Med., vol. 53, no. 1, pp. 1432-1440, Jun. 2005.

[12]M. Bertleff, S. Domsch, S. Weingärtner, et al. "Diffusion parameter mapping with the combined intravoxel incoherent motion and kurtosis model using artificial neural networks at 3T," NMR Biomed, 30:e383, Sep. 2017.

[13]G. Ertas, "Estimating the distributed diffusion coefficient of breast tissue in diffusion-weighted imaging using multilayer perceptrons," Soft Comput (2018). https://doi.org/10.1007/s00500-018-3412-6X

[14]C. Tamura, H. Shinmoto, S. Soga, et al. "Diffusion kurtosis imaging study of prostate cancer: Preliminary findings," J. Magn. Reson. Imaging, vol. 40, no. 3, pp. 723-729, Sep. 2014.

[15]S. Suo, X. Chen, W. Lianming, et al. "Non-Gaussian water diffusion kurtosis imaging of prostate cancer," Magn. Reson. Imaging, vol. 32, no. 5, pp. 421-427, Jun. 2014.

[16]M.C. Roethke, T.A. Kuder, T.H. Kuru, et al. "Evaluation of diffusion kurtosis imaging versus standard diffusion imaging for detection and grading of peripheral zone prostate cancer," Invest. Radiol., vol. 50 , no. 8, pp. 483-489, Aug. 2015

[17] T. Tamada, V. Prabhu, J. Li, et al. "Prostate cancer: diffusion-weighted MR imaging for detection and assessment of aggressivenesscomparison between conventional and kurtosis models," Radiology, vol. 284, no. 1, pp. 100-108, Jul. 2017.

[18]Z. Feng Z, X. Min X, D.J. Margolis, et al. "Evaluation of different mathematical models and different b-value ranges of diffusionweighted imaging in peripheral zone prostate cancer detection using bvalue up to $4500 \mathrm{~s} / \mathrm{mm} 2$," PLoS One, 15;12(2):e0172127, Feb. 2017.

[19]F. Langkilde, T. Kobus, A. Fedorov, et al. "Evaluation of fitting models for prostate tissue characterization using extended-range bfactor diffusion-weighted imaging," Magn. Reson. Med., vol. 79, no. 4, pp. 2346-2358, Apr. 2018.

[20] Y. Xi, A. Liu, F. Olumba, et al. "Low-to-high b value DWI ratio approaches in multiparametric MRI of the prostate: feasibility, optimal combination of $\mathrm{b}$ values, and comparison with ADC maps for the visual presentation of prostate cancer," Quant. Imaging Med. Surg., vol. 8, no. 6, pp. 557-567, Jul. 2018.

[21]Cho, G. Y. Cho, L. Moy, J.L. Zhang, et al. "Comparison of fitting methods and b-value sampling strategies for intravoxel incoherent motion in breast cancer," Magn. Reson. Med., vol. 74, no. 4, pp. 1077 1085, Oct. 2015.

[22]I. Jambor, H. Merisaari, P. Taimen, et al. "Evaluation of different mathematical models for diffusion-weighted imaging of normal prostate and prostate cancer using high b-values: a repeatability study," Magn. Reson. Med., vol. 73, no. 5, pp. 1988-98, May 2015.

[23]S. Chen, C.N. Cowan, P.M. Grant, "Orthogonal least squares learning algorithm for radial basis function networks," IEEE Trans. Neural Netw., vol. 2, no. 2, pp. 302-309, Mar. 1991.

[24]M.D. Pérez-Godoy, A.J. Rivera, C.J. Carmona, et al. "Training algorithms for radial basis function networks to tackle learning processes with imbalanced data-sets," Applied Soft Computing, vol. 25, pp. 26-39, Dec. 2014.

[25]P. Zhou and J. Austin, "Learning criteria for training neural network classifiers," Neural Comput. \& Applic., vol. 7, no. 4. pp. 334-342, 1998.

[26]J.D. Evans, Straightforward statistics for the behavioral sciences. Belmont, CA: Thomson Brooks/Cole, 1996.

[27]H. Lee, S.I. Hwang, H.J. Lee, et al. "Diagnostic performance of diffusion-weighted imaging for prostate cancer: Peripheral zone versus transition zone,” PLoS One, 13(6):e0199636, Jun 2018. 
[28]J.B. Gomm and D.L. Yu, "Selecting radial basis function network centers with recursive orthogonal least squares training," IEEE Trans. Neural Netw., vol 11, no. 2, pp. 306-314, Mar. 2000. 